Research Article

\title{
Improvement of N-Acetylcysteine Loaded in PLGA Nanoparticles by Nanoprecipitation Method
}

\author{
Ruth Lancheros $\mathbb{D}^{1},{ }^{1}$ Carlos A. Guerrero, ${ }^{2}$ and Rubén D. Godoy-Silva ${ }^{1}$ \\ ${ }^{1}$ Departamento de Ingeniería Química y Ambiental, Facultad de Ingeniería, Universidad Nacional de Colombia, \\ Bogotá, Colombia \\ ${ }^{2}$ Departamento de Ciencias Fisiológicas, Facultad de Medicina, Universidad Nacional de Colombia, Bogotá, Colombia \\ Correspondence should be addressed to Ruth Lancheros; rlancheros@unal.edu.co
}

Received 9 February 2018; Revised 21 May 2018; Accepted 7 June 2018; Published 5 July 2018

Academic Editor: Brajesh Kumar

Copyright (c) 2018 Ruth Lancheros et al. This is an open access article distributed under the Creative Commons Attribution License, which permits unrestricted use, distribution, and reproduction in any medium, provided the original work is properly cited.

\begin{abstract}
$\mathrm{N}$-Acetylcysteine (NAC) is a hydrophilic compound with a low bioavailability. It has been used as an effective antioxidant agent. This research seeks to enhance the entrapment of NAC in PLGA nanoparticles for drug delivery systems. The nanoparticles were made using the nanoprecipitation method and changing the following parameters: the solvent/nonsolvent nature, its viscosity, $\mathrm{pH}, \mathrm{NAC}$ addition to the nonsolvent, the polymer concentration and molecular weight, and NAC concentration in the solvent. The results showed that an increase in the nonsolvent viscosity produces NAC concentration in the solvent, and the nonsolvent rises its entrapment in the nanoparticles. Nanoparticles with $235.5 \pm 11.4 \mathrm{~nm}$ size with an entrapment efficiency of $0.4 \pm 0.04 \%$ and a specific load of $3.14 \pm 0.33 \%$ were obtained. The results suggest that besides efficiently entrapping hydrophobic compounds, the nanoprecipitation method also has a high potential as an alternative entrapment method for hydrophilic compounds as well. However, its use in the pharmaceutical industry, as a proper specific load vehicle, still depends on the improvement of the load capacity.
\end{abstract}

\section{Introduction}

$\mathrm{N}$-Acetylcysteine (NAC) is a mucolytic, anti-inflammatory, and hepaprotective agent used as a powerful antioxidant to protect cells $[1,2]$ and to treat diseases such as cancer, neuropsychiatric disorders, and cardiovascular diseases, among others [3,4]. Despite all these benefits, NAC has a low bioavailability (6 to $8 \%$ ), which limits its therapeutic effects. This happens because, once it enters the bloodstream, it joins the plasmatic proteins and creates disulfide bridges [5]. When supplied intravenously, nearly $30 \%$ of NAC is eliminated through, urine and high doses can increase the blood pressure $[5,6]$. Thus, the development of carriers to transport and stabilize NAC inside the body is a major concern when researching for new ways to increase its bioavailability. In this scenario, nanoparticles manufactured in materials such as PLGA, a biodegradable copolymer, are being developed [7-9].
Entrapping low molecular weight hydrophilic compounds, like NAC in PLGA nanoparticles, represents a challenge as the small size of the substance facilitates its migration and rapid release $[7,10]$. This study aims at increasing NAC entrapment in nanoparticles fabricated by the nanoprecipitation method, as it constitutes a simple, economic, and easily scalable methodology $[11,12]$. Even though this methodology is used mainly for liposoluble compounds, the literature presents successful cases of entrapment of hydrophilic compounds $[13,14]$. As shown in Figure 1, this method consists in dissolving the active compound (NAC) and the polymer (PLGA) in the same solvent. This solution is brought into contact with a different solvent (called nonsolvent), in which the polymer is insoluble. The first solvent migrates into the nonsolvent because the latter is completely miscible in the former. This results in the polymer precipitation that gives form to nanoparticles. Thus, in this process, nanoparticles 


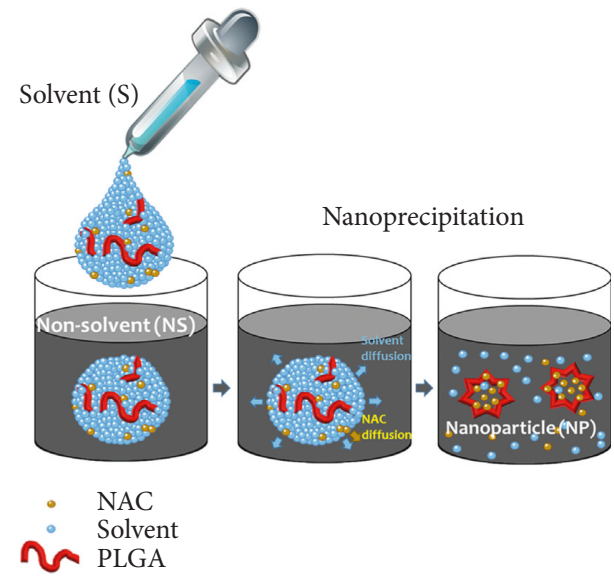

Figure 1: PLGA nanoparticle production scheme by nanoprecipitation methodology.

are expected to trap a considerable quantity of the active compound [15].

The quantity of the active compound that can be entrapped depends on factors such as the size of the molecules and the active compound solubility, as well as its affinity to the nonsolvent. If the active compound is highly soluble in the nonsolvent, it can migrate into the nonsolvent and reduce the quantity of compound trapped within the nanoparticles. Given that for medical purposes, the nanoparticles' diameter should not exceed 200 to $250 \mathrm{~nm}$ since a higher particle size can be retained by the reticuloendothelial system [16]. The strategies to increase the entrapment must focus on the two limiting conditions stated before.

There are multiple strategies reported in literature to enhance the entrapment of hydrophilic compounds in PLGA nanoparticles. For example, the nonsolvent, which is usually water, has been changed for another nonsolvent in which the active compound is less soluble. Some nonsolvents reported are caprylate and caprate triglyceride [17], cottonseed oil [13, 14], methanol, ethanol, and isopropanol [18]. It was found that cottonseed oil enhanced entrapment efficiency from 0.01 to $7.1 \%$ but increased the particle size $(300 \mathrm{~nm})$ and the polydispersity value $(0.4)$. The use of alcohols as nonsolvents affects the particle size, obtaining the smaller size using methanol $(102 \mathrm{~nm})$ and increasing it with the hydrocarbon chain length. Bilati et al. reported no values of entrapment efficiency [18]. Another method employed is to make the active compound more hydrophobic by modifying water $\mathrm{pH}$ when it is used as a nonsolvent. Tewes et al. used an alkali $\mathrm{pH}$ (8.6) to turn doxorubicin more hydrophobic, increasing the entrapment efficiency from $67 \%$ (using neutral $\mathrm{pH}$ ) to $95 \%$ [19]. Govender et al. reported similar results to enhance the entrapment of procaine hydrochloride from $11 \%$ using a $\mathrm{pH}$ of 5.8 to $58.5 \%$ with a $\mathrm{pH}$ value of 9.3 [20]. Peltonen et al. acidified the $\mathrm{pH}$ of sodium cromoglycate and enhanced the entrapment efficiency up to $70 \%$ [21]. Song and colleagues reported an increase of entrapment efficiency by changing the nonsolvent $\mathrm{pH}$ and increasing the molecular weight and the polymer concentration $[22,23]$.
The purpose was to develop a methodology to maximize the NAC entrapped inside PLGA nanoparticles $\left(C_{\mathrm{NAC}-\mathrm{S}}\right)$, minimize the migration of active compound towards the nonsolvent $\left(N_{\text {NAC-NS }}\right)$, and obtain a particle size under $250 \mathrm{~nm}$. Using Fick's law for compound transport [24], we sought to decrease both the diffusivity $\left(\mathscr{D}_{\text {NAC-NS }}\right)$ and the NAC concentration gradient between the inside and the outside of the nanoparticles $\left(\partial C_{\mathrm{NAC}-\mathrm{NS}} / \partial x\right)$.

As explained in Figure 2, we increased PLGA molecular weight, viscosity of the nonsolvent, and the PLGA concentration to decrease the diffusivity $\left(\mathscr{D}_{\mathrm{NAC}-\mathrm{NS}}\right)$. In this process, a variety of solvent/nonsolvent pairs were tested. The $\mathrm{pH}$ of the nonsolvent was varied and calcium ions were added to turn the active compound more hydrophobic. Additionally, NAC was added to the nonsolvent in order to decrease the concentration gradient $\left(\partial C_{\mathrm{NAC}-\mathrm{NS}} / \partial x\right)$ between the NAC solvent and nonsolvent.

\section{Materials and Methodology}

2.1. Materials. Polymers employed in the preparation of nanopaticles: PLGA 50:50 Resomer RG 502 (Mw 7-17 kDa), RG 504 (Mw 38-54 kDa), and RG 505 (Mw 54-69 kDa) ester terminated were purchased at Sigma-Aldrich.

Surfactants: Stepan-Mild L3 ${ }^{\circledR}$, Bio-Soft N1-7®, and Makon NF-5 ${ }^{\circledR}$ were acquired at Stepan. Pluronic F-127 ${ }^{\circledR}$ Bioreagent was acquired at Sigma-Aldrich.

2.1.1. Solvents. Dimethylsulphoxide R.A. (DMSO) and ethyl acetate R.A. were acquired at Panreac. Acetone and methyl acetate R.A. were purchased from Merck.

2.1.2. Nonsolvents. Type II water, $n$-butanol, $n$-heptane, and amyl acetate were obtained from Merck; propylene glycol from Dow; and Neobee ${ }^{\circledR} 1053$ from Stepan.

NAC handling and quantification: $\mathrm{FeCl}_{3} \cdot 6 \mathrm{H}_{2} \mathrm{O}$ and dichloromethane were purchased from Merck; 2,4,6-tris-(2pyridyl)-s-triazine (TPTZ) from Sigma-Aldrich; and $\mathrm{HCl}$ from J. Baker.

\subsection{Preparation of Nanoparticles}

2.2.1. Base Case. Nanoparticles formation was performed by the nanoprecipitation method using acetone as solvent and water as nonsolvent. $10 \mathrm{mg}$ of PLGA RG 502 and $2 \mathrm{mg}$ of NAC were dissolved in $1 \mathrm{~mL}$ of acetone in a glass vial. After the reactants' addition, the glass vial was immediately closed to prevent acetone evaporation. The vial was left for $2 \mathrm{~h}$ in dissolution at room temperature $\left(\sim 22^{\circ} \mathrm{C}\right)$ with sporadic shaking by manual inversion. Then, the solution was added manually $(\sim 1.5 \mathrm{ml} / \mathrm{min})$ into $10 \mathrm{ml}$ of water with Pluronic F-127 1\% (w/v) as surfactant, and magnetically stirred. The final suspension was held in stirring during 10 minutes, enough time to allow the solvent to migrate towards the water and form the nanoparticles.

From the base case, several modifications were done in order to increase the entrapment of NAC by the nanoparticles. Figure 2 illustrates the strategy used to minimize 


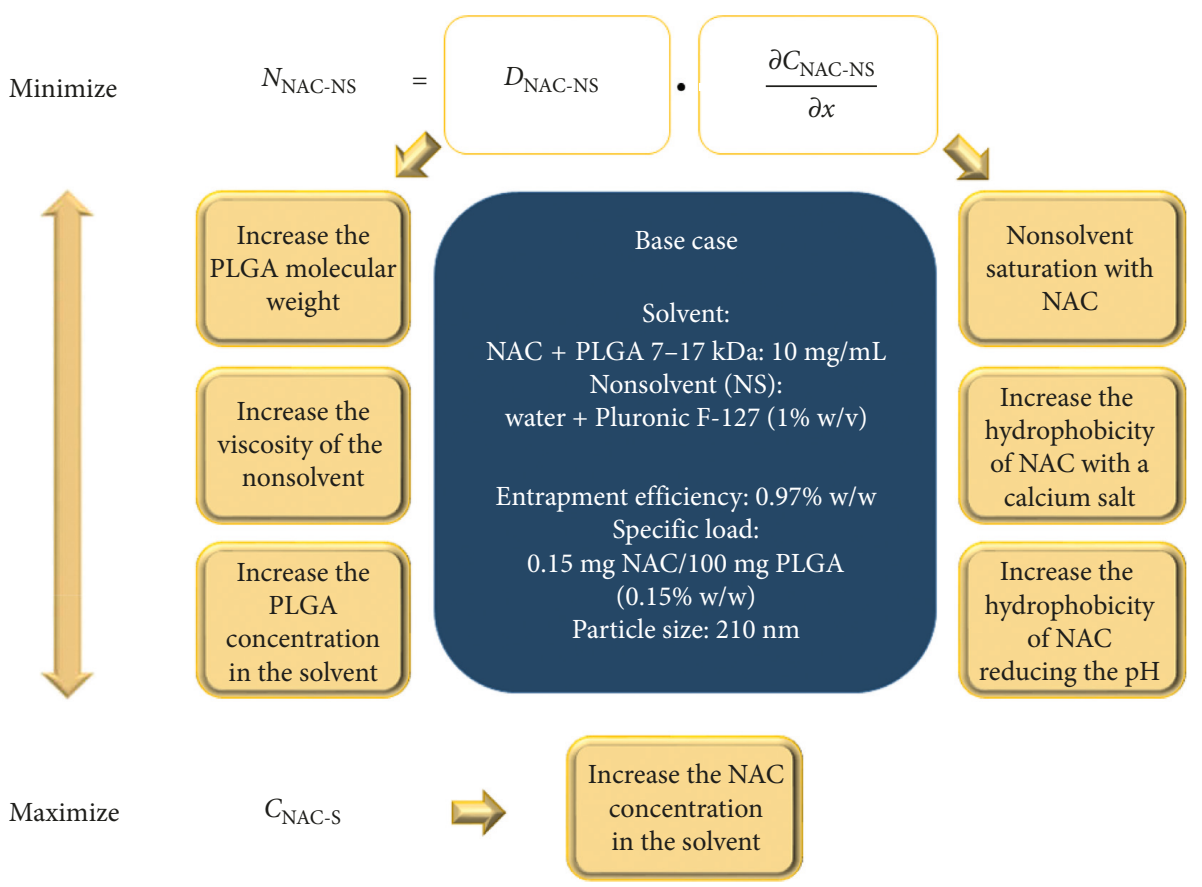

FIGURE 2: Strategies to increase the entrapped NAC in nanoparticles of PLGA.

TABLE 1: Solvent and nonsolvent effect on nanoparticle formation by nanoprecipitation method.

\begin{tabular}{|c|c|c|c|c|c|c|c|c|c|c|c|c|c|}
\hline \multirow{4}{*}{$\begin{array}{l}\text { Dielectric } \\
\text { constant }(\mathcal{E})\end{array}$} & \multirow{4}{*}{ Nonsolvent } & \multicolumn{12}{|c|}{ Particle size $(\mathrm{nm})$} \\
\hline & & & & & & & & & Solvent & & & & \\
\hline & & \multicolumn{3}{|c|}{$\begin{array}{l}\text { Ethyl acetate } \\
\qquad(\mathcal{E}=6.0)\end{array}$} & \multicolumn{3}{|c|}{$\begin{array}{l}\text { Methyl acetate } \\
\qquad(\varepsilon=6.7)\end{array}$} & \multicolumn{3}{|c|}{ Acetone $(\mathcal{E}=20.7)$} & \multicolumn{3}{|c|}{ DMSO $(\mathcal{E}=48.9)$} \\
\hline & & No & $\mathrm{AE}$ & $\mathrm{EL}$ & No & $\mathrm{AE}$ & EL & No & $\mathrm{AE}$ & EL & No & $\mathrm{AE}$ & EL \\
\hline 2.0 & Heptane & $\mathrm{A}$ & PS & PS & $\mathrm{A}$ & PS & PS & $\mathrm{A}$ & PS & PS & NM & NM & NM \\
\hline 3.0 & Neobee 1053 & $\mathrm{~A}$ & A & PS & $\mathrm{A}$ & AS & PS & A & $\mathrm{A}$ & PS & $\mathrm{NM}$ & NM & NM \\
\hline 5.0 & Amyl acetate & $\mathrm{A}$ & $\mathrm{A}$ & A & $\mathrm{A}$ & $\mathrm{A}$ & A & A & A & $\mathrm{A}$ & NM & NM & NM \\
\hline 17.1 & Butanol & A & A & A & $\mathrm{A}$ & $456.3 \pm 15.9$ & A & A & $287.7 \pm 5.6$ & A & $536.4 \pm 22.3^{*}$ & $524 \pm 22.2$ & A \\
\hline 32.0 & Propylene glycol & $\mathrm{A}$ & $\mathrm{A}$ & $\mathrm{A}$ & $\mathrm{A}$ & $\mathrm{A}$ & A & $\mathrm{A}$ & A & A & $696.8 \pm 13.6^{*}$ & $2772 \pm 861^{*}$ & $630.4 \pm 7.8^{*}$ \\
\hline
\end{tabular}

No: no surfactant used; AE: Bio-Soft N1-7; EL: Stepan-Mild L3; PS: partially soluble in the nonsolvent; A: aggregation; NM: not miscible; ${ }^{*}$ microparticles present in the suspension.

NAC gradient concentration and diffusivity. The following variables were considered:

(i) Solvent and nonsolvent identity: in the base case, we used acetone as the solvent and water as the nonsolvent with Pluronic F127 as a surfactant. As a strategy to avoid NAC diffusion into water, new couples different from acetone-water were used. The couples were selected according to four criteria: low toxicity (class 3 according to the specifications of the International Council for Harmonisation); miscibility; NAC insolubility in the nonsolvent; and similarity in the dielectric constant values (the higher the difference in the values of these constants, the higher will be the probability of nanoparticles aggregation [18]). Table 1 shows the different couples explored. The surfactant used in the base case was Pluronic F127, which is insoluble in all the nonsolvents used, so other surfactants were used. The surfactants chosen were nonionic to avoid reaction with NAC.

(ii) Nonsolvent viscosity: diffusivity is inversely proportional to nonsolvent viscosity. Thus, to increase its diffusivity, we added propylene glycol, which is a solvent with a viscosity of $48.6 \mathrm{cP}$ at $25^{\circ} \mathrm{C}$. At this temperature, NAC is slightly soluble. In the test, a mixture of equal volumes of propylene glycol and water $(6.12 \mathrm{cP})$ was used, and it was compared with the base case. In both cases, Pluronic F127 was added with a concentration of $1 \% \mathrm{w} / \mathrm{v}$.

(iii) Aqueous phase $\mathrm{pH}$ : NAC is protonated depending on the $\mathrm{pH}$ medium. The most hydrophobic form was reached at $\mathrm{pH}$ below 3.3 [25]. Tests were done at aqueous $\mathrm{pH}$ of 2.4 and 7.2. Given that NAC becomes unstable at higher levels, no tests at above pH 7.2 were done [26]. 
(iv) Solvent and nonsolvent ratio (S:NS) : different values were used: $1: 2,1: 3,1: 4,1: 5$, and $1: 10$. These values were derived from the literature [23, 27, 28].

(v) NAC concentration in the solvent: diverse values of NAC were used according to the solvent capacity to dissolve it. 5, 10, and $25 \mathrm{mg} / \mathrm{mL}$ (solubility at $25^{\circ} \mathrm{C} \sim 30 \mathrm{mg} / \mathrm{mL}$ ) were used for acetone, and $2,25,50,80$, and $100 \mathrm{mg} / \mathrm{mL}$ (solubility at $25^{\circ} \mathrm{C} \sim 100 \mathrm{mg} / \mathrm{mL}$ ) for were used DMSO.

(vi) NAC concentration in the nonsolvent: the nonsolvent was enriched with NAC in order to avoid its migration towards the nonsolvent by decreasing the concentration gradient. Thus, the concentration of NAC entrapped inside the nanoparticles increased. In this case, DMSO was used as solvent and NAC $(80 \mathrm{mg} / \mathrm{mL})$ was added to the nonsolvent (water + Pluronic F127).

(vii) Solvent type: the quantity of NAC available to be entrapped inside the nanoparticles is limited by its solubility in the solvent. Although the base case uses acetone, diverse tests were done with DMSO and the mixture $(1: 1)$ of acetone-DMSO, due to the high solubility of NAC in DMSO.

(viii) NAC-calcium salt formation: the neutralization of NAC with calcium hydroxide makes the molecule more hydrophobic [29]. The experiments were done adding the calcic salt of the $\mathrm{N}$-acetylcysteine (NAC-Ca) to the solvent or the calcium chloride ( 1 mol of $\mathrm{CaCl}_{2}: 2$ charged moles of NAC) to the nonsolvent (water + Pluronic F-127). The calciumNAC salt was prepared by blending the NAC solution in water $(90 \mathrm{mg} / \mathrm{mL})$ up to $\mathrm{pH} 6$ with calcium hydroxide $(0.165 \mathrm{w} / \mathrm{v} \%)$. Then, the mixture was lyophilized at $-47^{\circ} \mathrm{C}$ and $10 \mathrm{~Pa}$ pressure for $32 \mathrm{~h} \mathrm{[29].} \mathrm{In} \mathrm{this} \mathrm{assay,} \mathrm{the} \mathrm{base} \mathrm{case} \mathrm{solvent} \mathrm{was}$ not used because the NAC calcic salt is not soluble in acetone. Therefore, the solvent used was acetone-DMSO in a volumetric proportion of $1: 1$. As control, NAC was used in the solvent.

(ix) PLGA concentration: it has been reported that increasing the polymer concentration in the solvent rises the entrapment of the active compound and the particle size [30-32]. Even though the literature has reported values from 5 to $100 \mathrm{mg} / \mathrm{ml}$ [33-37], the PLGA concentrations used were 10 (base case) and $20 \mathrm{mg} / \mathrm{mL}$ since higher concentrations could increase particle size.

(x) Polymer molecular weight $(\mathrm{Mw})$ : this variable can affect both the releasing velocity and the entrapment efficiency of the active compound $[30,38]$. To evaluate this effect, different PLGA (50:50) polymers were used with molecular weight of 7$17 \mathrm{kDa}, 38-54 \mathrm{kDa}$, and $54-59 \mathrm{kDa}$.

The aim of this research was to obtained nanoparticles with the lowest particle size and the highest entrapment efficiency. Once all the variables were studied, a final assay was done using the better values found.

2.3. NACQuantification. The extraction of NAC was carried out with the centrifugation (Hermle Z $233 \mathrm{M}-2$ ) of $1 \mathrm{~mL}$ of nanoparticle aqueous suspension at $20,000 \mathrm{~g}$ for 20 minutes to discard the supernatant. Later, the pellet was washed three times adding $0.7 \mathrm{~mL}$ of type II water. To dissolve the resultant pellet, $400 \mu \mathrm{L}$ of DCM was added and the mixture was shaken with a vortex. The final solution was left at rest for 1 hour to guarantee the complete dissolution of the polymer. Afterward, $400 \mu \mathrm{L}$ of water was added. The mixture was shaken one more time and centrifuged at 20,000 $\mathrm{g}$ for 3 minutes.

The NAC quantification was done in the superior phase (aqueous) by the spectrophotometry method, using an adaptation of Kukoc and Radic's protocol $[39,40]$. For $60 \mu \mathrm{L}$ of the aqueous phase, a reactant was prepared with a solution of $1.2 \mathrm{~mL}$ of acetate buffer $(0.5 \mathrm{M}) \mathrm{pH} 3.6 ; 75 \mu \mathrm{L}$ of aqueous $\mathrm{Fe}$ (III) $(10 \mathrm{mM}) ; 75 \mu \mathrm{L}$ of aqueous solution of 2,4,6-tris-(2pyridyl)-s-triazine (TPTZ) $(10 \mathrm{mM})$; and $90 \mu \mathrm{L}$ of water. The final mixture obtained was shaken and kept for 60 minutes at room temperature until a purple color was achieved.

Finally, the absorbance value was quantified at $593 \mathrm{~nm}$ wavelength. The same treatment was used as blank with type II water instead of the sample. Concentration was determined using a calibration curve with detection limits ranging between 5 and $140 \mu \mathrm{g} / \mathrm{mL}$. The tests were carried out at least twice [40].

NAC entrapment efficiency (\%NAC) was determined by dividing the NAC mass entrapped inside the nanoparticles by its original added weight in the solvent solution and multiplying the result by 100 . The specific load $(\% \mathrm{w} / \mathrm{w}$ NAC/PLGA) was determined by dividing the NAC mass entrapped inside the nanoparticles by the added polymer mass in the solvent solution and multiplying the result by 100.

2.4. Determination of Particle Size, Polydispersity Index, and Zeta Potential. The particle size distribution was determined by the dynamic light scattering (DLS) with a Malvern Zetasizer ZS equipment at $25^{\circ} \mathrm{C}$ and a nanoparticle concentration of approximately $1 \mathrm{mg} / \mathrm{mL}$. The polydispersity index (PDI) is a parameter obtained simultaneously with the particle size. This index provides information about the sample: values close to 0 indicate that the sample was monodispersed, while values close to 1 indicate that the sample was polydispersed. Zeta potential was determined by laser Doppler velocimetry and phase analysis light scattering using the same equipment.

2.5. Nanoparticle Morphology. The nanoparticle morphology was studied using transmission electronic microscopy (TEM) employing an FEI equipment (Tecnai 20 Twin, $200 \mathrm{kV}$ ), with a $120 \mathrm{kV}$ voltage. 


\section{Results and Discussion}

The first assay done under the base case conditions obtained nanoparticles with an average diameter of $210 \pm 9 \mathrm{~nm}$ and PDI of $0.2 \pm 0.04$. The NAC entrapment efficiency was $0.97 \pm$ $0.2 \%$, and the specific load was $0.15 \pm 0.05 \%$. Even though the particle size satisfied the expected range, the results showed significantly low entrapment efficiency and specific load levels. These results justify the modifications implemented during the procedure.

3.1. Solvent and Nonsolvent Identity. The NAC is a compound with high solubility in water $(100 \mathrm{mg} / \mathrm{ml}[26])$, which means that in this case, the use of water as nonsolvent would reduce its entrapment. A strategy to increase it was to replace water with another nonsolvent completely miscible with the organic solvent. In turn, the new nonsolvent should not solubilize NAC, so, several couples of solvents and nonsolvents were tested (Table 1). The results showed aggregation in the couples with low dielectric constant. Similar results were obtained by Dalpiaz et al. who experimented with acetone (solvent) and cottonseed oil (nonsolvent) to obtain nanoparticles with $200 \mathrm{~nm}$ size and a relatively high polydispersity index ranging between 0.3 and 0.4 . Conversely, the results obtained from the DMSO and propylene glycol couple without surfactant showed particle sizes of $696.8 \mathrm{~nm}$ with a polydispersity index of 0.13 . It is worth mentioning that both solvents have the highest dielectric constants ( 48.9 and 32, resp.). Although particles with the desired size were not achieved (below $250 \mathrm{~nm}$ ), higher sizes and monodispersions were obtained (PDI $<0.250$ ). This appears attractive for other applications, as preventing osteoblasts death in orthopedics applications by incorporating the particles into an acrylic bone cement [41], as an intravesical delivery system to treat bladder diseases [42], or in dryer power inhaler [43] among others.

3.2. Nonsolvent Viscosity. Figure 3(a) shows the variation of particle size, efficiency, and specific load with the increase of the nonsolvent viscosity. As you can see, when the nonsolvent (propylene glycol/water $50: 50$ ) viscosity augmented, the particle size increased slightly $(10 \%)$. This probably happened because the nanoparticle formation mechanism is given by the migration of the solvent towards to the nonsolvent, which turns the polymer insoluble. When water is enriched with propylene glycol, which is also a polar solvent completely miscible with acetone, the formation mechanism is not altered, showing similar results. In fact, the NAC solubility in water and in water with propylene glycol, it presents no considerable change. Regarding the retained NAC, both the entrapment efficiency and specific charge increased 4 and 3 times, respectively. In this research, we proposed that the higher the medium viscosity, the lower the diffusion of NAC from the acetone polymer solution towards the aqueous phase.
3.3. Aqueous Phase pH. Figure 3(b) shows the effect of the aqueous phase $\mathrm{pH}$ over particle size, efficiency, and specific load. Monodispersed nanoparticles with sizes below $250 \mathrm{~nm}$ were capable of entrapping NAC in a low percentage. The particle size increased in $18 \%$, the entrapment efficiency in $17 \%$, and the specific load in $18 \%$. Although a higher entrapment of NAC and bigger particles at $\mathrm{pH} 2.4$ than in the same test at neutral $\mathrm{pH}$ were observed, this difference could be found in the experimental error. Generally, no significant difference was found either in the entrapment (efficiency and specific charge) or the particle size. This indicates that even though the acid $\mathrm{pH}$ makes NAC molecule hydrophobic, the difference is not considerable. Similar results have been reported by Song et al. [23], who varied aqueous phase $\mathrm{pH}$ while entrapping vinicristine and found that at $\mathrm{pH}$ higher than 7 , the entrapment increases by $10 \%$ (approximately). It was also found that the particle size remains constant.

3.4. Solvent and Nonsolvent Ratio (S:NS). The influence of this variable in all the variable responses considered (\%NAC, specific load, and particle size) can be observed in Figure 3(c). The particle size increases when a smaller volume is added (S: NS $1: 2$ ). This occurs because part of the solvent remains in the polymeric matrix, increasing its size. With higher volumes of nonsolvent, the particle size decreases and almost remains constant since $1: 3$. Additionally, entrapment efficiency and specific charge decrease while the aqueous phase volume rises. This is a consequence of the increment in the concentration gradient between the solvent and the nonsolvent. Similar results were reported by other authors [21, 24, 27]. Since low size nanoparticles and high entrapment efficiency are observed, it is concluded that the best ratio S:NS would be $1: 3$. Thus, for further tests, this ratio would be preferred.

3.5. NAC Concentration in the Solvent. The available amount of NAC to be entrapped varies according to the solvent capacity to dissolve it. In the case of acetone, the added concentration cannot be higher than $30 \mathrm{mg} / \mathrm{mL}$ (at room temperature $\approx 22^{\circ} \mathrm{C}$ ), because at higher concentrations, the solubilization of the compound was not possible. However, the concentration used was higher $(100 \mathrm{mg} / \mathrm{mL})$ with DMSO.

Figures 3(d) and 3(e) show that the particle size does not change significantly for any of the solvents used, and it is not affected by the NAC added. Conversely, it can be affirmed that the active compound does not interfere in the nanoparticle formation because its particle size remains constant, even with higher concentrations $(100 \mathrm{mg} / \mathrm{mL})$. The efficiency values are low. However, this behavior is different when it comes to the specific charge since it increases with the NAC amount added and gives a higher quantity of molecules to retain inside the polymeric matrix. The increment of the specific charge is even clearer in DMSO than in acetone, given that the polymer faces a higher amount of NAC. These results indicate that the polymeric matrix can retain a higher number of molecules inside when the quantity of available 


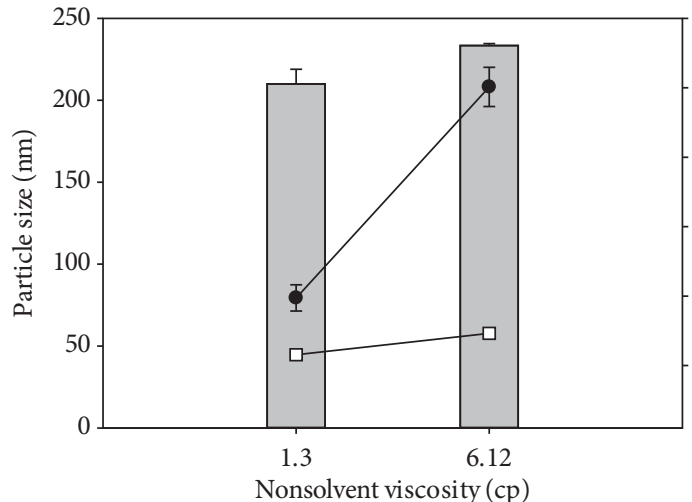

(a)

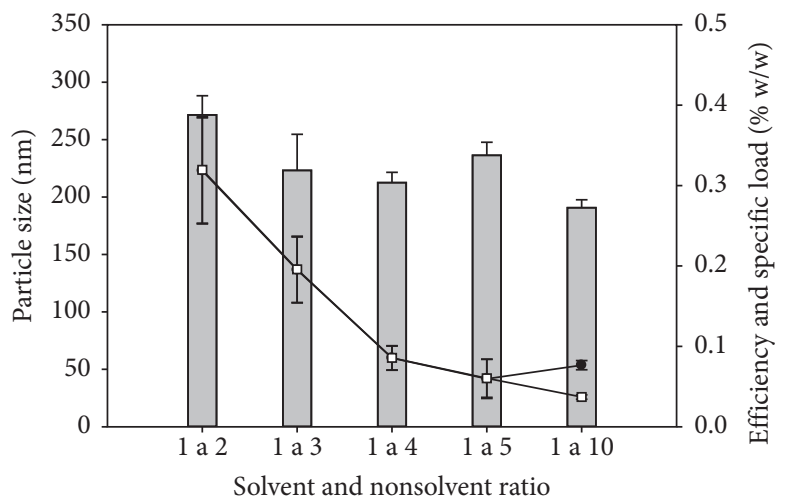

(c)

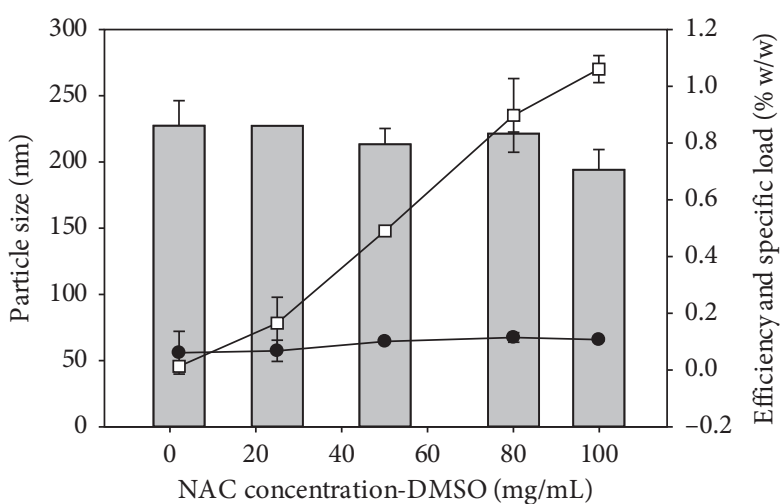

(e)

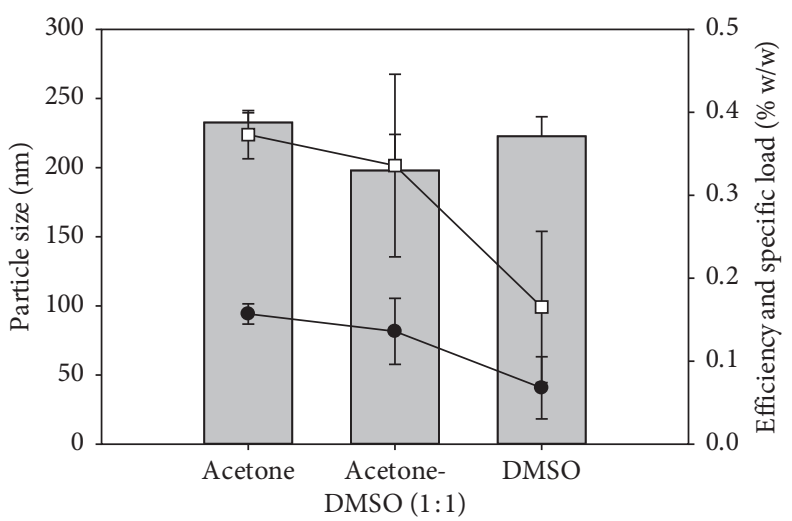

(g)

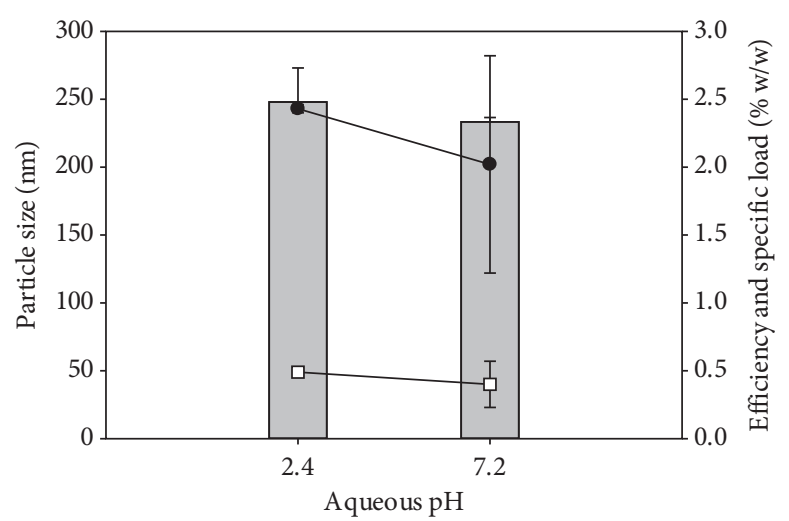

(b)

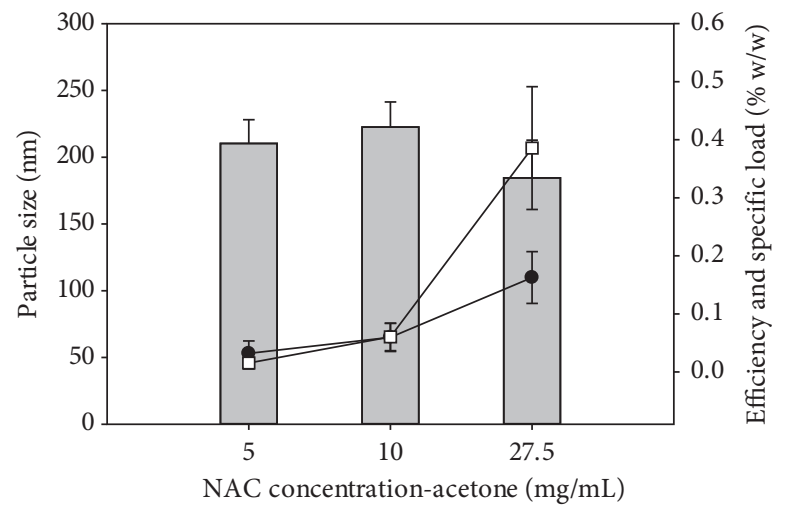

(d)

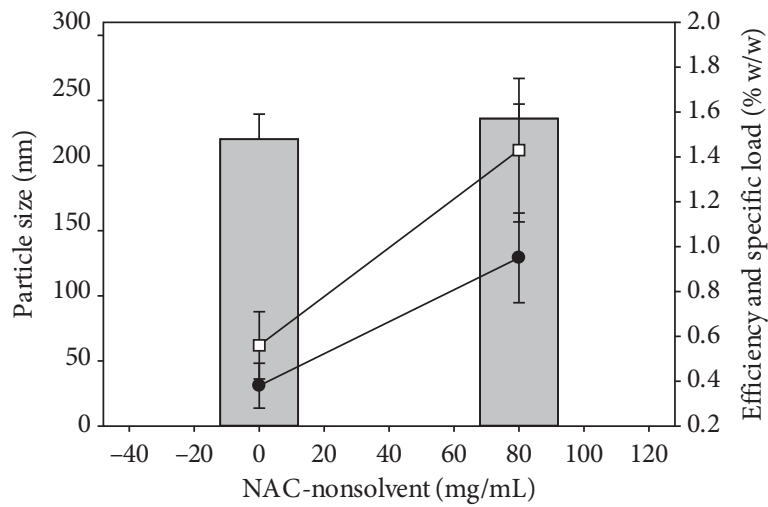

(f)

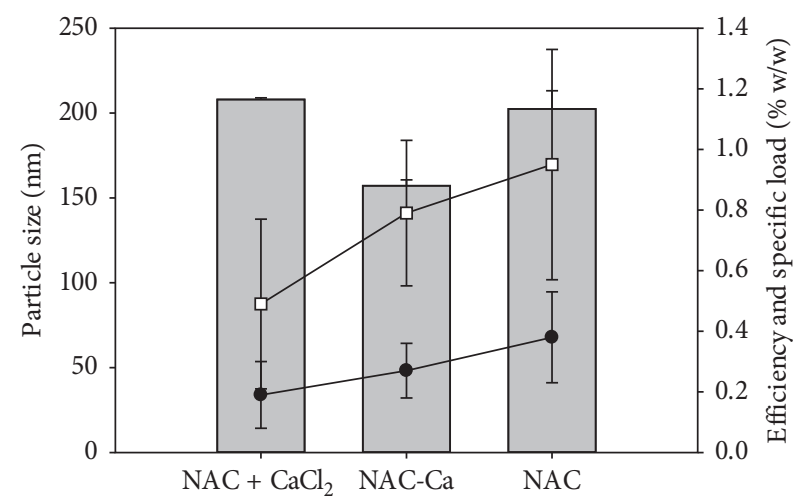

(h)

Figure 3: Continued. 


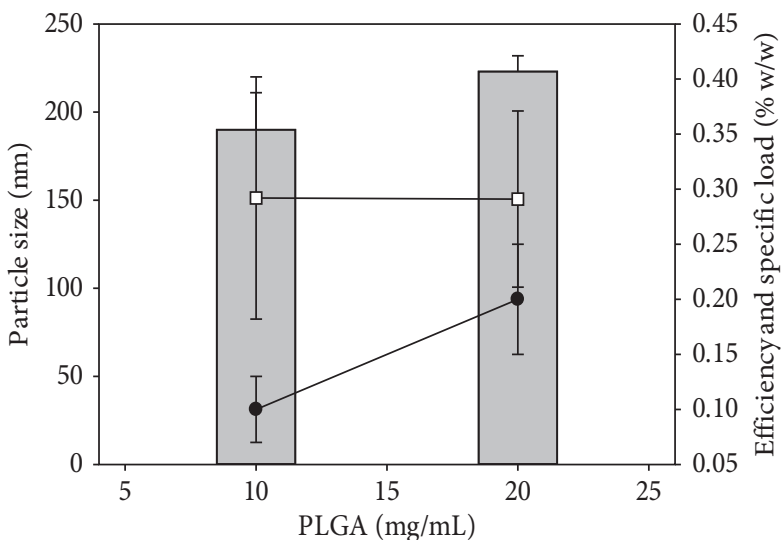

(i)

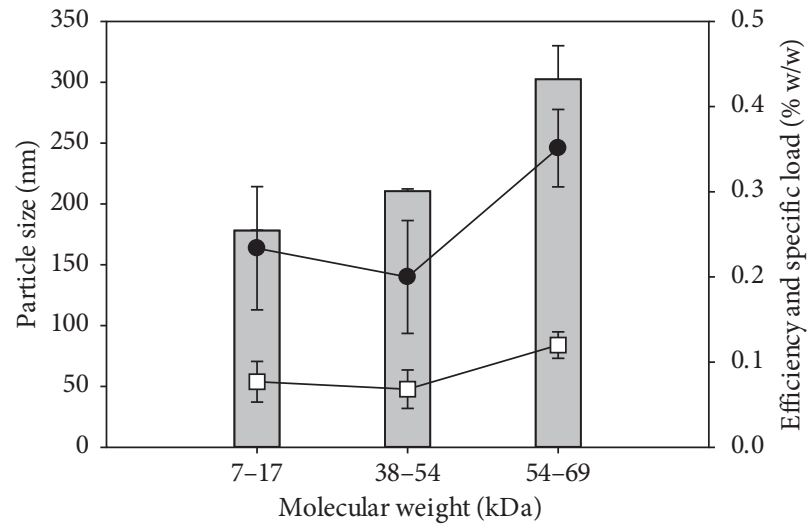

(j)

Figure 3: Effects of different variables on the average particle size ( $\square$ ), entrapment efficiency ( $\bullet-)$, and specific load ( $\neg-)$ of PLGA nanoparticles loaded with NAC. (a) Nonsolvent viscosity, (b) aqueous phase $\mathrm{pH}$, (c) solvent and nonsolvent ratio, (d) NAC concentration in acetone, (e) NAC concentration in DMSO, (f) NAC concentration in nonsolvent, (g) solvent type, (h) NAC-calcium salt, (i) polymer concentration, and (j) polymer molecular weight.

molecules is higher. Other researchers report similar results using different solvents $[44,45]$.

3.6. NAC Concentration in the Nonsolvent. In Figure 3(f), the particle size variation, the entrapment efficiency, and the specific load when the NAC is added to the nonsolvent can be observed. The particle size increased slightly (7\%), while the entrapment efficiency and the specific load increased 2.5 times approximately. This indicates that the particle size is not significantly affected by the presence of NAC in the nonsolvent, probably due to the low entrapment. In addition, efficiency and specific load increased notably by the NAC presence in the nonsolvent inside the nanoparticles, which may result from the diminishing of the NAC concentration gradient.

Other authors report the addition of active compound to the nonsolvent as a strategy to increase the entrapment $[46,47]$. For example, Avgoustakis et al. report similar values for cisplatin entrapment, which increases from $0.1 \%$ to $0.66 \%$ when saturating the nonsolvent [46]. Although NAC entrapment increases effectively when adding NAC to the nonsolvent (as much as twice), the NAC-enriched aqueous phase will not be used until the final test in order to describe the behavior of other variables.

3.7. Solvent Type. Different tests were done using DMSO, acetone, and a mixture $(1: 1)$ of both substances that have low-grade toxicity (class 3 in ICH classification). The aim of the tests with the mixture of solvents was to increase the acetone capacity of solubilizing NAC, considering that they complement each other. DMSO has proven to solubilize NAC in concentrations up to $100 \mathrm{mg} / \mathrm{ml}$, but it presents a higher trend to agglomerate when resuspended after centrifugation. On the contrary, the particles produced with acetone remain in suspension. This can be verified when comparing the zeta potential $(-33.4 \mathrm{mV}$ for acetone and $-9.8 \mathrm{mV}$ for DMSO).
Figure $3(\mathrm{~g})$ shows that the change of solvent does not affect the particle size, which achieved values ranging between 200 and $250 \mathrm{~nm}$. Similar results including surfactants have been reported. For example, Song et al. found that when surfactant is employed, the particle size remains quasi constant [45].

Regarding efficiency and specific charge, a decrement when DMSO is used as solvent was observed. However, such a decrease is observed in acetone and in the mixture of solvents. This allows concluding that the use of a mixture of solvents would be the best option since it allows increasing the amount of NAC that can be added to the solvent, and its separation process by centrifugation would be less susceptible to agglomeration.

3.8. NAC-Calcium Salt Formation. The results of Figure 3(h) show the effect of adding calcium to NAC in order to make it more hydrophobic. When using the NAC salt, it is observed that the particle size decreases almost to $25 \%$, but the efficiency and the specific charge are higher in the samples of NAC without calcium. These results suggest that the presence of calcium ions in the molecule of NAC does not affect the entrapment efficiency. These results contrast to those reported by Desai et al. [29], who improve the entrapment efficiency from $87.9 \%$ to $98.4 \%$ using salt of NAC-Ca, although the entrapment methodology for the active component is different.

3.9. PLGA Concentration. Results on Figure 3(i) show the effect of increasing the polymer concentration, regarding the particle size, the entrapment efficiency, and the specific load. As you can see, the particle size increased in $17 \%$, while the entrapment efficiency increased almost twice, but the specific load remains without any variation. This happened because the polymer quantity added increased as well as the particle size; therefore, there is a higher capacity to entrap 
the active compound inside the nanoparticles. As the specific load is related to the amount of added polymer, and it is increased, the final result is that the specific load remains constant. Similar results have been reported by other researchers [21, 27]. Mora-Huertas et al. report a bigger particle size than the one used in this research [31]. For this reason, the lowest concentration was chosen since it produces smaller particle size and the same specific load.

3.10. Polymer Molecular Weight. Figure 3(j) shows the effect of polymer molecular weight on the particle size, the entrapment efficiency, and the specific load. The particle size changes with the molecular weight. However, for values $7-17 \mathrm{kDa}$ and $38-54 \mathrm{kDa}$, the change is negligible $(18 \%$ compared to the value obtained for $7-17 \mathrm{kDa}$ ), while for the highest molecular weight $(54-69 \mathrm{kDa})$ it increased almost $70 \%$. Regarding the entrapment efficiency and the specific load, they both increased slightly with the increasing molecular weight, particularly with the higher value (both the entrapment efficiency and the specific load increased almost $50 \%$, compared to the data obtained for $7-17 \mathrm{kDa}$ ). Other researchers have reported an increase in the efficiency and particle size with the molecular weight [23, 38, 48]. This indicates that the increase in the particle size happens due to the increase in the polymeric chain length. This would explain the slight increase for both the entrapment efficiency and specific load. It would be necessary to determine the variation of the pore size inside the nanoparticles with the change of molecular weight, to verify whether it is affected or not. Inasmuch as more porous particles are obtained, it would be easier for the compound to abandon the polymeric matrix.

3.10.1. Final Assay. Table 2 shows the effect of the variables studied on the particle size, the efficiency, and specific load. Accordingly, a final assay was proposed using the best found conditions. Water with propylene glycol $(1: 1)$ and Pluronic F127 was used as nonsolvent (surfactant $1 \% \mathrm{w} / \mathrm{v}$ ); this nonsolvent was enriched with NAC $(80 \mathrm{mg} / \mathrm{mL})$. As solvent, a mixture of acetone and DMSO $(1: 1)$ with an NAC concentration of $80 \mathrm{mg} / \mathrm{ml}$ and $10 \mathrm{mg} / \mathrm{ml}$ of PLGA $38-54 \mathrm{kDa}$ was used. As a result, spherical shape nanoparticles were obtained, which did not present aggregation (Figure 4 ). The histogram (Figure 5(a)) shows the different particle sizes obtained by TEM, and the Figure 5(b) shows the graph obtained by DLS. Based on micrographs of TEM, particle sizes vary from 76 to $180 \mathrm{~nm}$ with an average of $111.6 \pm 24.8$ $(\mathrm{n}=43)$. These results differ from values obtained by DLS $(235.5 \pm 11.4 \mathrm{~nm})$. It could be explained by the fact that TEM samples were dried as a preparation for TEM imaging, while DLS reported the hydrodynamic diameter that includes core plus any molecule attached on surface; this kind of difference between these techniques had been reported by other researchers $[49,50]$. The nanoparticle zeta potential was $-3.42 \pm 0.5 \mathrm{mV}$, the entrapment efficiency was $0.4 \pm 0.04 \%$, and the specific load was $3.14 \pm 0.33 \%$. The latter increased almost 20 times compared with the data obtained for the base case. As a comparison, other research has reported
TABLE 2: Effect of variables on particle size, entrapment efficiency, and specific load.

\begin{tabular}{lccc}
\hline Variable & $\begin{array}{c}\text { Particle } \\
\text { size }\end{array}$ & Efficiency & $\begin{array}{c}\text { Specific } \\
\text { load }\end{array}$ \\
\hline Viscosity nonsolvent & $=$ & + & + \\
$\mathrm{pH}$ & $=$ & $=$ & $=$ \\
O/V volume ratio & - & - & - \\
Concentration NAC solvent & $=$ & $=$ & + \\
Concentration NAC & $=$ & + & + \\
nonsolvent & $=$ & $=$ & $=$ \\
Solvent & - & $=$ & $=$ \\
Ca addition & + & + & $=$ \\
PLGA concentration & + & $=$ & $=$ \\
PLGA molecular weight & & &
\end{tabular}

higher and lower values for the specific load using similar molecules, which were made by the nanoprecipitation method: $\mathrm{Lu}$ et al. found $21 \%$ for doxirubicin trapped in mPEG-PLGA particles [51], Dalpiaz et al. reported a 7.4\% for protamine [14], Govender et al. found 4.1\% for Procaine loaded in PLGA nanoparticles [20], Yang et al. reported $2.59 \%$ for doxorubicin loaded in PEG-PLA particles [52], and Dalpiaz et al. found $0.26 \%$ for $\mathrm{N}^{6}$-cyclopentyladenosine. PLGA-NAC nanoparticles have also been obtained by other methods like electrospray with a drug load of $5 \%$ [53].

It is necessary to keep optimizing the process in order to obtain higher entrapments and increase the vehicle transport capacity. As a possibility, the NAC size could be increased, by joining this molecule to a bigger one and forming a bond that can be broken by an enzyme in the human body. Other alternative could be to find a solvent that generates smaller pores in order to increase the nanoparticle density and restrict the NAC migration to the nonsolvent or modify the copolymer adding a molecule to make the polymer more hydrophilic.

\section{Conclusions}

Different alternatives were evaluated in order to increase the entrapment of a hydrophilic low molecular weight compound such as NAC inside PLGA nanoparticles. It was found that the particle size was not affected by the nonsolvent viscosity, the type of solvent, or the NAC concentration in the solvent or nonsolvent. However, the ratio between the solvent and nonsolvent (S:NS), the polymer concentration and molecular weight, the use of calcium salt, and the use of different solvent-nonsolvent couples influence the particle size. It was also found that most of the solventnonsolvent couples tested presented agglomeration, except those formed by acetone and DMSO (solvents) with propylene glycol and butanol (nonsolvent), which have a high dielectric constant.

The specific charge and the entrapment efficiency were favored by the increase in the nonsolvent viscosity, the NAC concentration in the solvent, and the enrichment of nonsolvent with NAC. However, both of them decreased with higher volumes of nonsolvent (S:NS). 


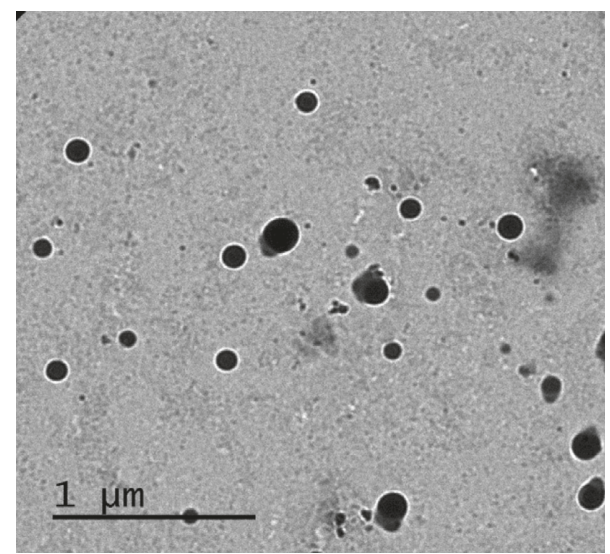

(a)

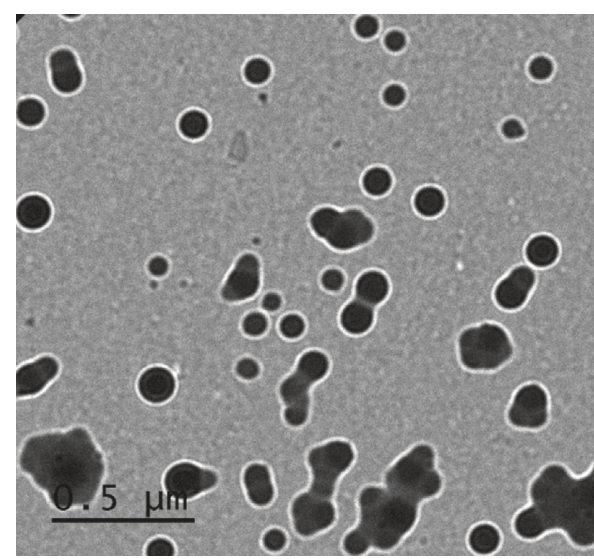

(b)

FIgURE 4: Transmission electron micrographs of PLGA-NAC nanoparticles.

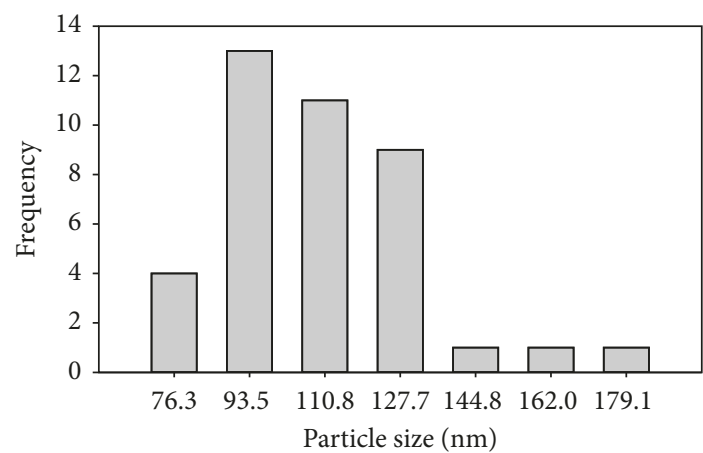

(a)

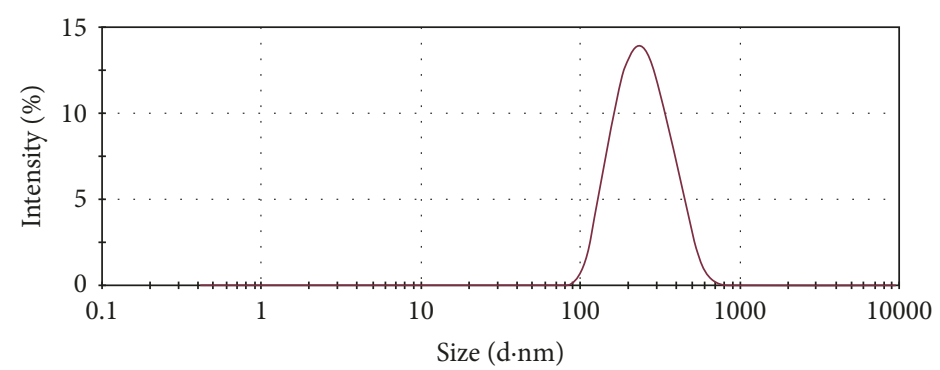

(b)

FIGURE 5: (a) Histogram with the distribution of particle size measure by TEM. (b) Distribution of particle size obtained by DLS.

A methodology that allows the NAC entrapped inside PLGA nanoparticles to increase, keeping the particle size below $250 \mathrm{~nm}$ and a specific load of 3.1\% (20 times higher than the base case). Nevertheless, before using other strategies to increase the entrapment, different tests should be done in order to evaluate whether the current methodology affects or not the NAC biological activity.

\section{Data Availability}

All the data are available in http://www.bdigital.unal.edu. co/55610/13/RuthJannethLancherosSalas.2016.pdf.

\section{Conflicts of Interest}

The authors declare that they have no conflicts of interest.

\section{Acknowledgments}

This research was funded by Dirección de Investigación of Bogotá de la Universidad Nacional de Colombia Grant 202010018338 of 2012 and by Departamento Administrativo de Ciencia, Tecnología e Innovació-COLCIENCIAS Grant 110156935147.

\section{References}

[1] H. Bulut and G. H. Sayar, "N-acetylcysteine in treatment of trichotillomania," Journal of Neurobehavioral Sciences, vol. 2, no. 3, p. 110, 2015.

[2] L. Xue, J. Li, Y. Li et al., "N-acetylcysteine protects Chinese hamster ovary cells from oxidative injury and apoptosis induced by microcystin-LR," International Journal of Clinical and Experimental Medicine, vol. 8, no. 4, pp. 4911-4921, 2015.

[3] M. Zafarullah, W. Q. Li, J. Sylvester, and M. Ahmad, "Molecular mechanisms of $\mathrm{N}$-acetylcysteine actions," Cellular and Molecular Life Sciences (CMLS), vol. 60, no. 1, pp. 6-20, 2003.

[4] S. Naveed, A. Amray, A. Waqas, A. M. Chaudhary, and M. W. Azeem, "Use of $\mathrm{N}$-acetylcysteine in psychiatric conditions among children and adolescents : a scoping review," Cureus, vol. 9, no. 11, 2017.

[5] R. S. Navath, Y. E. Kurtoglu, B. Wang, S. Kannan, R. Romero, and R. M. Kannan, "Dendrimer-drug conjugates for tailored intracellular drug release based on glutathione levels," Bioconjugate Chemistry, vol. 19, no. 12, pp. 2446-2455, 2008.

[6] B. Wang, R. S. Navath, R. Romero, S. Kannan, and R. Kannan, "Anti-inflammatory and anti-oxidant activity of anionic dendrimer-N-acetyl cysteine conjugates in activated microglial cells," International Journal of Pharmaceutics, vol. 377, no. 1-2, pp. 159-168, 2009.

[7] F. Ramazani, W. Chen, C. F. Van Nostrum et al., "Strategies for encapsulation of small hydrophilic and amphiphilic drugs 
in PLGA microspheres: state-of-the-art and challenges," International Journal of Pharmaceutics, vol. 499, pp. 358-367, 2016.

[8] S. Sharma, A. Parmar, S. Kori, and R. Sandhir, "PLGA-based nanoparticles: a new paradigm in biomedical applications," TrAC Trends in Analytical Chemistry, vol. 80, pp. 30-40, 2016.

[9] S. Chou, S. Gunaseelan, M. Hussam et al., "A review of injectable and implantable biomaterials for treatment and repair of soft tissues in wound healing," Journal of Nanotechnology, vol. 2017, Article ID 6341710, 15 pages, 2017.

[10] S. Vrignaud, J.-P. Benoit, and P. Saulnier, "Strategies for the nanoencapsulation of hydrophilic molecules in polymer-based nanoparticles," Biomaterials, vol. 32, no. 33, pp. 8593-604, 2011.

[11] H. A. Almoustafa, M. A. Alshawsh, and Z. Chik, "Technical aspects of preparing PEG-PLGA nanoparticles as carrier for chemotherapeutic agents by nanoprecipitation method," International Journal of Pharmaceutics, vol. 533, no. 1, pp. 275-284, 2017.

[12] C. J. Martínez Rivas, M. Tarhini, W. Badri et al., "Nanoprecipitation process: from encapsulation to drug delivery," International Journal of Pharmaceutics, vol. 532, no. 1, pp. 66-81, 2017.

[13] A. Dalpiaz, E. Vighi, B. Pavan, and E. Leo, "Fabrication via a nonaqueous nanoprecipitation method, characterization and in vitro biological behavior of n 6-cyclopentyladenosineloaded nanoparticles," Journal of Pharmaceutical Sciences, vol. 98, no. 11, pp. 4272-4284, 2009.

[14] A. Dalpiaz, F. Sacchetti, A. Baldisserotto et al., "Application of the "in-oil nanoprecipitation" method in the encapsulation of hydrophilic drugs in PLGA nanoparticles," Journal of Drug Delivery Science and Technology, vol. 32, pp. 283-290, 2016.

[15] K. Avgoustakis, "Pegylated poly(lactide) and poly(lactide-coglycolide) nanoparticles: preparation, properties and possible applications in drug delivery," Current Drug Delivery, vol. 1, no. 4, pp. 321-333, 2004.

[16] D. E. Owens and N. Peppas, "Opsonization, biodistribution, and pharmacokinetics of polymeric nanoparticles," International Journal of Pharmaceutics, vol. 307, no. 1, pp. 93102,2006

[17] H. Takeuchi, H. Yamamoto, and Y. Kawashima, "Mucoadhesive nanoparticulate systems for peptide drug delivery," Advanced Drug Delivery Reviews, vol. 47, no. 1, pp. 39-54, 2001.

[18] U. Bilati, E. Allémann, and E. Doelker, "Development of a nanoprecipitation method intended for the entrapment of hydrophilic drugs into nanoparticles," European Journal of Pharmaceutical Sciences, vol. 24, no. 1, pp. 67-75, 2005.

[19] F. Tewes, E. Munnier, B. Antoon et al., "Comparative study of doxorubicin-loaded poly(lactide-co-glycolide) nanoparticles prepared by single and double emulsion methods," European Journal of Pharmaceutics and Biopharmaceutics, vol. 66, no. 3 , pp. 488-492, 2007.

[20] T. Govender, S. Stolnik, M. C. Garnett, L. Illum, and S. S. Davis, "PLGA nanoparticles prepared by nanoprecipitation: drug loading and release studies of a water soluble drug," Journal of Controlled Release, vol. 57, no. 2, pp. 171-185, 1999.

[21] L. Peltonen, J. Aitta, S. Hyvönen, M. Karjalainen, and J. Hirvonen, "Improved entrapment efficiency of hydrophilic drug substance during nanoprecipitation of poly(l)lactide nanoparticles," AAPS PharmSciTech, vol. 5, no. 1, pp. 115-120, 2004.

[22] X. Song, Y. Zhao, W. Wu et al., "PLGA nanoparticles simultaneously loaded with vincristine sulfate and verapamil hydrochloride: systematic study of particle size and drug entrapment efficiency," International Journal of Pharmaceutics, vol. 350, no. 1-2, pp. 320-329, 2008.

[23] X. Song, Y. Zhao, S. Hou et al., "Dual agents loaded PLGA nanoparticles: systematic study of particle size and drug entrapment efficiency," European Journal of Pharmaceutics and Biopharmaceutics, vol. 69, no. 2, pp. 445-453, 2008.

[24] T. Higuchi, "Mechanism of sustained-action medication," Journal of Pharmaceutical Sciences, vol. 52, no. 12, pp. 1145-1149, 1963.

[25] B. Noszál, D. Visky, and M. Kraszni, "Population, acid - base, and redox properties of $\mathrm{N}$-acetylcysteine conformers," Journal of Medicinal Chemistry, vol. 43, no. 11, pp. 2176-2182, 2000.

[26] S. Aldrich Sigma, Data Sheet, N-Acetyl-L-Cysteine, 2012.

[27] J. Cheng, B. A. Teply, I. Sherifi et al., "Formulation of functionalized PLGA-PEG nanoparticles for in vivo targeted drug delivery," Biomaterials, vol. 28, no. 5, pp. 869-876, 2007.

[28] F. Danhier, N. Lecouturier, B. Vroman et al., "Paclitaxelloaded PEGylated PLGA-based nanoparticles: in vitro and in vivo evaluation," Journal of Controlled Release, vol. 133, no. 1, pp. 11-17, 2009.

[29] K. Desai, S. Mallery, and S. Schwendeman, "Formulation and characterization of injectable poly(-lactide-co- glycolide) implants loaded with N-acetylcysteine, a MMP inhibitor," Pharmaceutical Research, vol. 25, no. 3, pp. 586-597, 2008.

[30] A. Budhian, S. J. Siegel, and K. I. Winey, "Haloperidol-loaded PLGA nanoparticles: systematic study of particle size and drug content," International Journal of Pharmaceutics, vol. 336, no. 2, pp. 367-375, 2007.

[31] C. E. Mora-Huertas, H. Fessi, and A. Elaissari, "Influence of process and formulation parameters on the formation of submicron particles by solvent displacement and emulsification-diffusion methods. Critical comparison," Advances in Colloid and Interface Science, vol. 163, no. 2, pp. 90-122, 2011.

[32] H.-Y. Kwon, J.-Y. Lee, S.-W. Choi, Y. Jang, and J.-H. Kim, "Preparation of PLGA nanoparticles containing estrogen by emulsification-diffusion method," Colloids and Surfaces A: Physicochemical and Engineering Aspects, vol. 182, no. 1-3, pp. 123-130, 2001.

[33] G. Dalwadi and B. Sunderland, "An ion pairing approach to increase the loading of hydrophilic and lipophilic drugs into PEGylated PLGA nanoparticles," European Journal of Pharmaceutics and Biopharmaceutics, vol. 71, no. 2, pp. 231-242, 2009.

[34] A. Beletsi, Z. Panagi, and K. Avgoustakis, "Biodistribution properties of nanoparticles based on mixtures of PLGA with PLGA-PEG diblock copolymers," International Journal of Pharmaceutics, vol. 298, no. 1, pp. 233-241, 2005.

[35] V. Saxena, M. Sadoqi, and J. Shao, "Indocyanine green-loaded biodegradable nanoparticles: preparation, physicochemical characterization and in vitro release," International Journal of Pharmaceutics, vol. 278, no. 2, pp. 293-301, 2004.

[36] E. Cenni, D. Granchi, S. Avnet et al., "Biocompatibility of poly (D,L-lactide-co-glycolide) nanoparticles conjugated with alendronate," Biomaterials, vol. 29, no. 10, pp. 1400-1411, 2008.

[37] F. N. Costa, A. L. Ibiapino, L. P. de Figueiredo et al., "Preliminary evaluation of the encapsulation of new antidiabetic sulphonylhydrazone and antitumor $N$-acylhydrazone derivatives using PLGA nanoparticles," Journal of Physics: Conference Series, vol. 617, article 012015, 2015.

[38] R. C. Mundargi, V. R. Babu, V. Rangaswamy, P. Patel, and T. M. Aminabhavi, "Nano/micro technologies for delivering 
macromolecular therapeutics using poly(D,L-lactide-coglycolide) and its derivatives," Journal of Controlled Release, vol. 125, no. 3, pp. 193-209, 2008.

[39] L. Kukoc-Modun and N. Radić, "Kinetic spectrophotometric determination of $\mathrm{N}$-acetyl-L-cysteine based on a coupled redox-complexation reaction," Analytical Sciences, vol. 26, no. 4, pp. 491-495, 2010.

[40] L. Kukoc-Modun and N. Radić, "Spectrophotometric determination of N-acetyl-L-cysteine and N-(2-mercaptopropionyl)glycine in pharmaceutical preparations," International Journal of Analytical Chemistry, vol. 2011, Article ID 140756, 6 pages, 2011.

[41] H. Aita, N. Tsukimura, M. Yamada et al., "N-acetyl cysteine prevents polymethyl methacrylate bone cement extractinduced cell death and functional suppression of rat primary osteoblasts," Journal of Biomedical Materials Research Part A, vol. 92, no. 1, pp. 285-296, 2009.

[42] N. Denora, A. Lopedota, M. Perrone et al., "Spray-dried mucoadhesives for intravesical drug delivery using $\mathrm{N}$-acetylcysteine-and glutathione-glycol chitosan conjugates," Acta Biomaterialia, vol. 43, pp. 170-184, 2016.

[43] M. D. Manniello, P. Del, R. P. Aquino, and P. Russo, "Clarithromycin and $\mathrm{N}$-acetylcysteine co-spray-dried powders for pulmonary drug delivery: a focus on drug solubility," International Journal of Pharmaceutics, vol. 533, no. 2, pp. 463-469, 2017.

[44] B. Sinha, B. Mukherjee, and G. Pattnaik, "Poly-lactide-coglycolide nanoparticles containing voriconazole for pulmonary delivery: in vitro and in vivo study," Nanomedicine: Nanotechnology, Biology and Medicine, vol. 9, no. 1, pp. 94104, 2013.

[45] K. C. Song, H. S. Lee, I. Y. Choung, K. I. Cho, Y. Ahn, and E. J. Choi, "The effect of type of organic phase solvents on the particle size of poly(d,l-lactide-co-glycolide) nanoparticles," Colloids and Surfaces A: Physicochemical and Engineering Aspects, vol. 276, no. 1-3, pp. 162-167, 2006.

[46] K. Avgoustakis, A. Beletsi, Z. Panagi, P. Klepetsanis, A. G. Karydas, and D. S. Ithakissios, "PLGA-mPEG nanoparticles of cisplatin: in vitro nanoparticle degradation, in vitro drug release and in vivo drug residence in blood properties," Journal of Controlled Release, vol. 79, no. 1-3, pp. 123-135, 2002.

[47] D. Moreno, S. Zalba, I. Navarro, C. Tros de Ilarduya, and M. J. Garrido, "Pharmacodynamics of cisplatin-loaded PLGA nanoparticles administered to tumor-bearing mice," European Journal of Pharmaceutics and Biopharmaceutics, vol. 74, no. 2, pp. 265-274, 2010.

[48] G. Mittal, D. K. Sahana, V. Bhardwaj, and M. N. V. Ravi Kumar, "Estradiol loaded PLGA nanoparticles for oral administration: effect of polymer molecular weight and copolymer composition on release behavior in vitro and in vivo," Journal of Controlled Release, vol. 119, no. 1, pp. 77-85, 2007.

[49] E. Yoo, Y. Liu, C. A. Nwasike et al., "Surface characterization of nanoparticles using near-field light scattering," Beilstein Journal of Nanotechnology, vol. 9, pp. 1228-1238, 2018.

[50] K. L. Douglas and M. Tabrizian, "Effect of experimental parameters on the formation of alginate-chitosan nanoparticles and evaluation of their potential application as DNA carrier," Journal of Biomaterials Science, Polymer Edition, vol. 16, no. 1, pp. 43-56, 2005

[51] P. Lu, Y. Chen, T. Ou et al., "Multifunctional hollow nanoparticles based on graft-diblock copolymers for doxorubicin delivery," Biomaterials, vol. 32, no. 8, pp. 2213-2221, 2011.
[52] Q. Yang, L. Tan, C. He et al., "Redox-responsive micelles selfassembled from dynamic covalent block copolymers for intracellular drug delivery," Acta Biomaterialia, vol. 17, pp. 193-200, 2015.

[53] A. Akbar, K. Zarchi, S. Abbasi et al., "Development and optimization of $\mathrm{N}$-acetylcysteine-loaded poly (lactic-coglycolic acid) nanoparticles by electrospray," International Journal of Biological Macromolecules, vol. 72, pp. 764-770, 2015. 


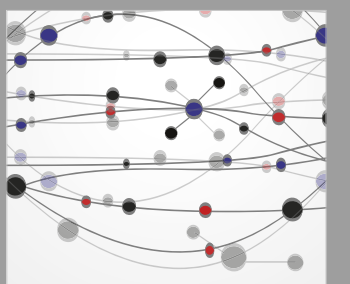

The Scientific World Journal
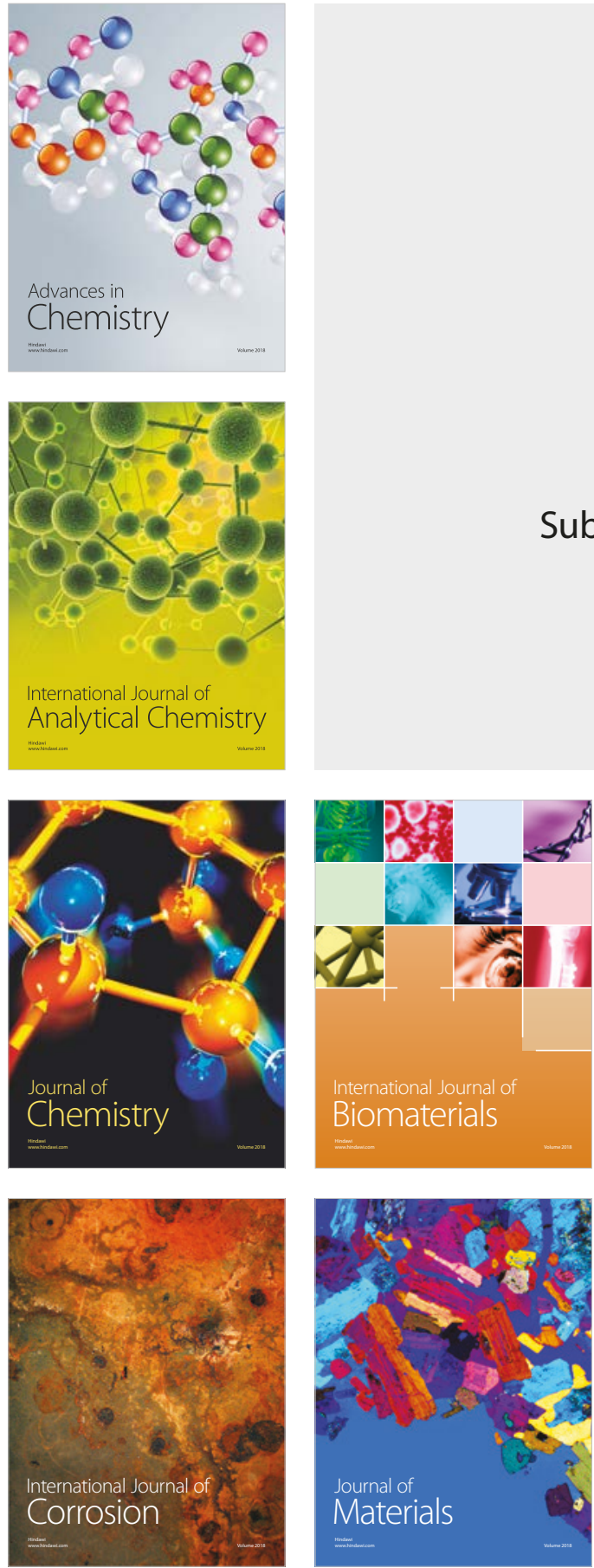

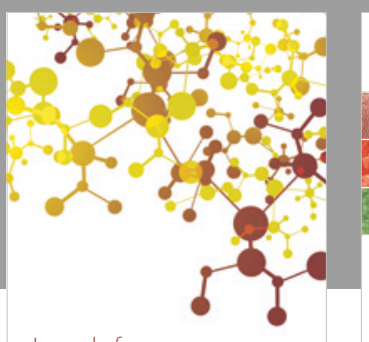

Journal of

Applied Chemistry
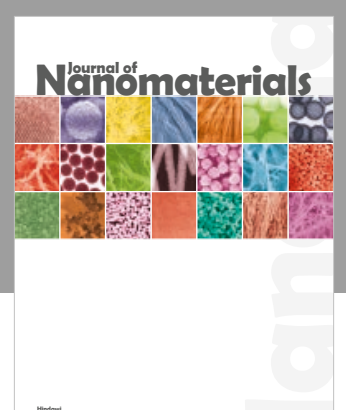

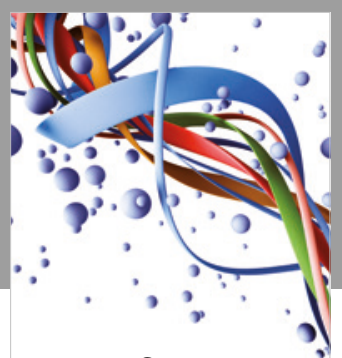

Scientifica

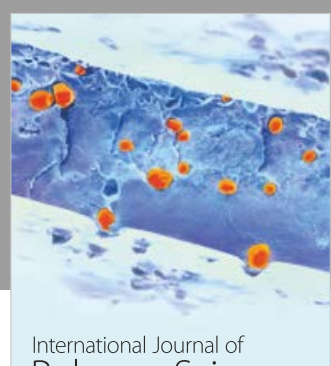

Polymer Science

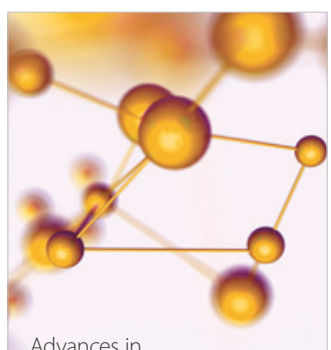

Physical Chemistry
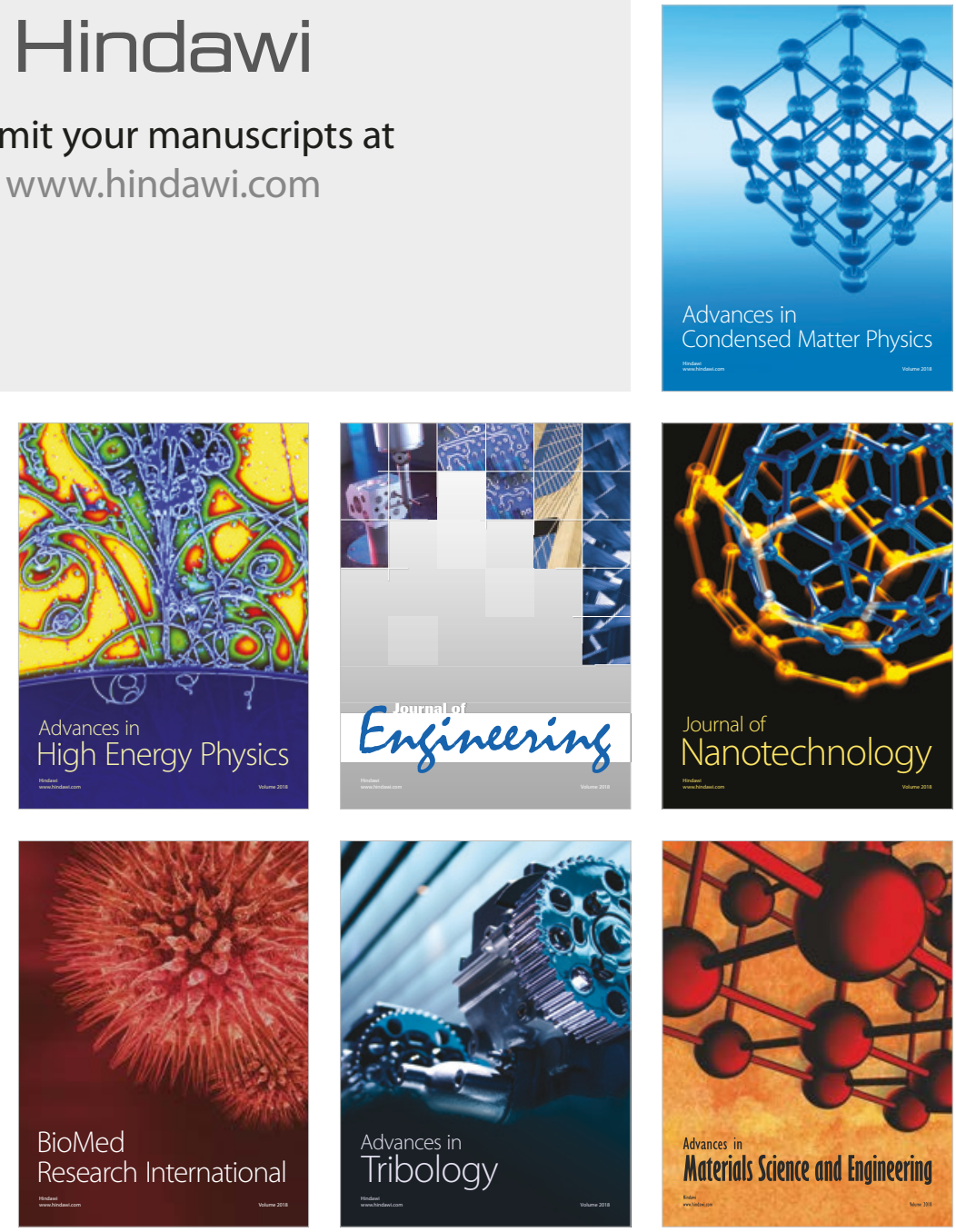